Article

\title{
Incorporation of Tannic Acid in Food-Grade Guar Gum Fibrous Mats by Electrospinning Technique
}

\author{
Weiqiao Yang ${ }^{1,2}$, Min Zhang ${ }^{1}$, Xihong Li ${ }^{1, *}$, Jianan Jiang ${ }^{1}$, Ana M.M. Sousa ${ }^{3}$, Qiang Zhao ${ }^{4}$, \\ Sherri Pontious ${ }^{5}$ and LinShu Liu ${ }^{3}$ (B) \\ 1 State Key Laboratory of Food Nutrition and Safety, School of Food Engineering and Biotechnology, \\ Tianjin University of Science and Technology, Tianjin 300457, China; yangweiqiaospring@126.com (W.Y.); \\ zm0102@tust.edu.cn.com (M.Z.); jiangjianan111@126.com (J.J.) \\ 2 Tianjin Jiesheng Donghui Fresh-keeping Technology Co., Ltd, Tianjin 300403, China \\ 3 Dairy and Functional Foods Research Unit, United States Department of Agriculture, Agricultural Research \\ Service, Eastern Regional Research Center, 600 East Mermaid Lane, Wyndmoor, PA 19038, USA; \\ ana.sousamm@gmail.com (A.M.M.S.); LinShu.Liu@ARS.USDA.GOV (L.S.L.) \\ 4 State Key Laboratory of Medicinal Chemical Biology, Key Laboratory of Bioactive Materials, Ministry of \\ Education, College of Life Sciences, Nankai University, Tianjin 300071, China; qiangzhao@nankai.edu.cn \\ 5 Department of Microbiology and Immunology, Temple University School of Medicine, Philadelphia, \\ PA 19140, USA; sherriannpontious@gmail.com \\ * Correspondence: lixihong@tust.edu.cn; Tel.: +86-139-2047-0396
}

Received: 20 December 2018; Accepted: 10 January 2019; Published: 15 January 2019

\begin{abstract}
The use of polysaccharides to produce functional micro- or nanoscale fibrous mats has attracted growing interest for their food-grade applications. In this study, the characterization and electro-spinnability of guar gum (GG) solutions loaded with tannic acid (TA) was demonstrated. Food-grade antioxidant materials were successfully produced by electrospinning while incorporating different loads of TA into GG fibers. Bead-free GG-TA fibers could be fabricated from GG solution ( $2 \mathrm{wt} \%$ ) with $10 \mathrm{wt} \% \mathrm{TA}$. Increasing the amount of TA led to fibers with defects and larger diameter sizes. Fourier Transformed Infrared Spectroscopy and X-ray Diffraction of neat GG and TA loaded GG fibrous mats suggested that inclusion of TA interrupted the hydrogen bonding and that a higher density of the ordered junction zones formed with the increased TA. The high TA incorporation efficiency and retained antioxidant activity of the fibrous mats afford a potential application in active edible film or drug delivery system.
\end{abstract}

Keywords: electrospinning; guar gum; tannic acid; antioxidant; nanofiber

\section{Introduction}

The interrelationship between structure and physicochemical characteristics for delivery and encapsulation materials requires the design to employ three key characteristics: (1) matrices with high porous structure and huge surface area-volume ratio to permit medium, relatively free exchange between cellular constructs, due to low permeability resistance [1,2]; (2) morphology-controlled process and toxic reagent-free methods, due to potential human and environmental safety concerns; and (3) greater incorporation of instructive molecules such as food additives or drugs to offer significant opportunities for enhancing the functionality [3].

Among various methods, the electrospinning technique has attracted growing interest to produce such materials and proven to be a relatively efficient and versatile nanotechnology to develop continuous fibers with diameters down to nano- to micrometer dimensions [4-6], which afford inherently high surface area-volume ratios and have excellent mass transfer properties [7]. Functional or unstable agents can be encapsulated with these fibers by mixing therapeutic additives with biopolymers when 
spinning, with improved bioavailability and controlled release [8-10]. In terms of functional delivery system, the microstructure has shown an important impact on digestion and absorption of bioactive compounds in the digestive tract [11,12]. However, most of the reported nanofibers are fabricated from synthetic polymers, in which organic solvents were commonly used to dissolve polymers, it is still challenging to produce $100 \%$ food-grade nanofibers using natural materials such as polysaccharides.

Guar gum (GG), which is one type of galactomannan acquired from the seeds of Cyamopsis psoraloides, is a naturally occurring hydrocolloid polysaccharides [13,14]. Due to its natural nontoxic, biodegradable, and cost-effective properties, GG has been considered a substitute for starch in food industry because this dietary polysaccharide is admittedly resistant to human digestion and absorption, with cholesterol and glucose lowering effects $[15,16]$. It also has been widely employed as food additives to affect the physicochemical properties of various food products such as ice cream, bakery products, beverages, etc. [17]. Oral administration using GG as the delivery system for drugs show that GG could release active agent in the colon and delay their release in target site under conditions mimicking mouth to gastrointestinal tract [18]. Hence, it occupies superior position in the food industry as a functional ingredient owing to its dietary fiber characteristic [19-21], as well as in the pharmaceutical field for drug delivery systems [22,23], which makes it an ideal carrier candidate to encapsulate a variety of bioactive food ingredients. The potential for electrospun GG has not yet been fully realized due to its high viscosity at a comparatively low content. The poor spinnability of GG is owing to it supra-molecular aggregate structures consisting of galactomannan molecules that interact with each other through hydrogen bonding [24,25]. Some researchers have used gums to generate nanofibrous polysaccharides-based materials for biomedical applications, but synthetic biopolymers (e.g., polyvinyl alcohol) are still needed as a carrier to overcome the poor fiber forming ability [26,27]. Although purification and filtration processes are used to improve the morphology [25], it is still hard to develop the continuous uniform nanostructure of GG fiber, much less use it to deliver functional additives.

Tannic acid (TA) is a natural phenolic compound that is ubiquitously distributed in an abundance of fruits and plants. Because of its ability to inhibit the hydroxyl radical formation by providing an electron or hydrogen atom, numerous studies have shown that TA exhibits excellent antioxidant, anticarcinogenic, and antimicrobial properties [28-30]. These biological advantages make TA a promising option for the fabrication of functional additives [30,31]. The plant-derived components (GG and TA) are both recognized as safe by FDA and even edible. The use of natural polysaccharides to develop active materials has a wide potential application in drug delivery systems [7], functional foods [32] and active edible packaging [33].

In this study, we examined the feasibility of incorporation of TA to develop electrospun GG fibrous mats with antioxidant activity for the first time. Prior to electrospinning, GG molecular entanglement in fiber formation was investigated. Next, $100 \%$ food-grade antioxidant materials were successfully produced by electrospinning, while incorporating different loads of TA into GG fibers. For the resultant fibrous mats, incorporation of TA in the fiber formation and physical characteristics were examined, including morphology, size, crystallinity, thermal stability, antioxidant activity, etc. The use of an efficient and organic solvent-free electrospinning method to fabricate edible functional GG fibrous mats demonstrates the potential for green electrospinning and its food-grade applications.

\section{Materials and Methods}

\subsection{Materials}

Tannic acid (TA, $\mathrm{C}_{76} \mathrm{H}_{52} \mathrm{O}_{46}$ ) and commercial guar gum (GG, molecular weight of $\mathrm{Mw}=3.896 \times 10^{6} \mathrm{~g} / \mathrm{mol}$ ) were obtained from Sigma-Aldrich (Saint Louis, MO, USA). Ethanol (ACS grade) was obtained from Tianjin Sixth Chemical LTD (Tianjin, China). 1,10-diphenyl-2-picrylhydrazyl (DPPH) was purchased from TCI Shanghai (Shanghai, China). All aqueous solutions were prepared with MilliQ water (resistivity of $18.2 \mathrm{M} \Omega$ ) from a Barnstead E-pure water system (Dubuque, IA, USA). 


\subsection{Preparation of GG and GG-TA Spinning Solution}

\subsubsection{Purification of GG}

Commercial GG needed to be purified before use to remove impurities existing in the powder. The purification of GG was according to Lubambo's report by ethanol abstraction method with some modification [25]. In brief, the GG was slowly dispersed into a vigorous vortex of Millipore water $(\sim 1.3 \mathrm{wt} \%)$ at room temperature and continuously stirred $(700 \mathrm{rpm} / \mathrm{min})$ overnight using a magnetic stirrer until complete polymer dissolution was achieved. The mixture was then transferred into the centrifuge tubes and centrifuged at $40{ }^{\circ} \mathrm{C}$ at $8000 \mathrm{rpm} / \mathrm{min}$ for $40 \mathrm{~min}$. A $500 \mathrm{~mL}$ dripping funnel was used to contain the supernatant and control its slow precipitation into ethanol at a 1:2 volume ratio. This process was under continuous stirring until the completion of the precipitation. The obtained white particles were collected and washed with ethanol three times followed by suction filtration, and then dried for $24 \mathrm{~h}$ at room temperature in a vacuum oven. The resulting pure GG was ground into powder for further use.

\subsubsection{Preparation of GG and GG-TA Spinning Solutions}

A series of GG solutions with different concentrations (0.6-2.2 wt \%) were prepared by dispersing a certain amount of the purified GG powder into water at $50{ }^{\circ} \mathrm{C}$. To completely dissolve the GG, the mixtures were incubated overnight at the same temperature with gentle magnetic stirring $(700 \mathrm{rpm} / \mathrm{min})$. To study the effects of incorporation of TA on fiber formation, four solutions varying by $5 \%$ of TA loaded GG spinning solution were prepared prior to electrospinning. These solutions were designated as GG-TA $5 \%$, GG-TA $10 \%$, GG-TA $15 \%$, and GG-TA $20 \%$, respectively. They were prepared by mixing precise amounts of TA with concentrations ranging from 5 to $20 \mathrm{wt} \%$ (based on the weight of dry GG) with adequate GG spinning solutions. The mixtures were constantly stirred $(1000 \mathrm{rpm} / \mathrm{min})$ at $50{ }^{\circ} \mathrm{C}$ for $3 \mathrm{~h}$ using a magnetic stirrer until homogeneous mixtures were obtained.

\subsection{Viscosity Measurements}

Viscous behavior was analyzed using the shear rheology test and performed through a Kinexus Rheometer (Malvern Instruments, Worcestershire, UK) by using an attached cone-plate geometry (diameter $40 \mathrm{~cm}, 2^{\circ} \theta$, truncation gap $54 \mu \mathrm{m}$ ). The prepared GG solutions ranging from 0.6 to $2.2 \mathrm{wt} \%$ were carefully loaded on the Peltier stage (preheated to $50^{\circ} \mathrm{C}$ ) and subsequently covered with paraffin oil to prevent the solution from drying. The apparent viscosity of the sample, responding to steady shear flow was analyzed with increasing shear rate from 0.001 to $1000 \mathrm{~s}^{-1}$.

The Cross-Williamson model was used to calculate the zero-shear viscosity $\left(\eta_{0}\right)$ from the flow curves as the following equation according to previous report [34]:

$$
\frac{\eta_{\text {app }}}{\eta_{0}}=\frac{1}{1+k \dot{\gamma}^{(1-v)}}
$$

where $\eta_{\text {app }}$ represents the apparent viscosity, $\eta_{0}$ represents the zero-shear viscosity, $k$ is the time constant, $\gamma$ represents the shear rate, and $\mathrm{n}$ indicates the flow index.

By using the $\eta_{0}$, the specific viscosity $\left(\eta_{\mathrm{sp}}\right)$ was obtained by the following formula [35,36]:

$$
\eta_{\mathrm{sp}}=\frac{\eta_{0}-\eta_{\mathrm{s}}}{\eta_{\mathrm{s}}}
$$

where $\eta_{\mathrm{s}}$ is the solvent viscosity.

\subsection{Electrospinning}

The electrospinning process was carried out on a YF SP-T unit (Yunfan Technologies Co., Limited, Shenzhen, China). Approximately $3 \mathrm{~mL}$ of the composite solution were loaded onto a $5 \mathrm{~mL}$ plastic 
syringe, attached with a stainless-steel needle placed in the spinneret. The conducting needle had an outer diameter of $0.7 \mathrm{~mm}$ and inner diameter of $0.40 \mathrm{~mm}$. Through a series of pretests under various process parameters, the optimum electrospinning condition in this work was fixed at: applied voltage, $18 \mathrm{kV}$; distance tip-to-collector, $10 \mathrm{~cm}$; feed rate $0.5 \mathrm{~mL} / \mathrm{h}$; and drum rotating speed, $40 \mathrm{rpm} / \mathrm{min}$. For all cases, the electrospinning temperature was set at $50{ }^{\circ} \mathrm{C}$ to prevent the formation of gel when the solutions were ejected from the tip of spinneret. After continuously electrospinning for $6 \mathrm{~h}$, fibrous mats were collected on the aluminum foil that covered the surface of the drum. The obtained mats were stored at controlled relative humidity (53\%) for $24 \mathrm{~h}$ before being tested.

\subsection{Characterization of Nanofibers}

\subsubsection{Scanning Electron Microscopy (SEM)}

Prior to imaging, samples were fixed on the specimen stubs by double sided tape and then coated with gold sputter. The morphology of the nanofibers was characterized through a 0182-S Phenom-World Scanning Electron Microscope (SEM, Thermo Fisher Scientific, Pittsburgh, PA, USA) following our previously reported procedure [31,37]. The images were acquired with the working condition of the instrument set at: accelerating voltages, $10 \mathrm{kV}$; high-vacuum/secondary electron imaging mode; and working distance, 10.1-11.4 mm. The average fiber diameters were analyzed using ImageJ Software v1.45 (NIH, Bethesda, MD, USA). The mean values of the fiber diameters, along with standard deviations of distributions, were determined after measuring 200 fiber diameters at random.

\subsubsection{Incorporation Efficiency of $\mathrm{TA}$}

The TA content in the solution was analyzed using the spectrophotometric method according to our previous report [31,37], and the incorporation efficiency (IE) of TA in the fibers was obtained based on the following equation:

$$
\mathrm{IE} \%=\frac{\text { Actual TA concentration }}{\text { Theoretical TA concentration }} \times 100 \%
$$

\subsubsection{Fourier Transformed Infrared Spectroscopy Analysis}

The infrared spectra of the GG-TA fibrous mats were examined using a Nicolet iS50 Fourier transform infrared (FT-IR) spectroscopy (Thermo Scientific, Pittsburgh, PA, USA) with a diamond Attenuated Total Reflectance (ATR) system attached. The fibrous mats were scanned at $0-4000 \mathrm{~cm}^{-1}$ wavelength and OMNIC (spectral signal processing software) was used to obtain the transmittance curve of the sample at different wavelengths.

\subsubsection{X-Ray Diffraction (XRD) Measurements}

The XRD patterns were recorded using a Philips Analyzer PW 1830 X-ray VB (1.54 A, Philips, Amedo, the Netherlands) with $\mathrm{Cu}-\mathrm{K} \alpha$ radiation. Scans were obtained in the $2 \theta$ range with a step size of $0.01^{\circ}$ per $1 \mathrm{~s}$. The scanning rate was set at $2^{\circ} / \mathrm{min}$.

\subsubsection{Thermal Properties}

Differential scanning calorimetry (DSC) was carried out on the pure GG powder and the as-spun electrospun fibrous mats by a DSC 204 F1 system (Netzsch, Germany). The thermal analysis for all samples $(2.5 \mathrm{mg})$ were performed at a $10^{\circ} \mathrm{C} / \mathrm{min}$ heating rate with increasing temperature from 50 to $250{ }^{\circ} \mathrm{C}$ in nitrogen.

\subsubsection{Antioxidant Activity}

The antioxidant activity of the fibrous membranes was measured by means of 1,10-diphenyl-2-picrylhydrazyl (DPPH), as reported by Neo et al. [25]. Briefly, a $10 \mathrm{mg}$ fiber membrane 
was accurately submerged in a tube containing $10 \mathrm{~mL}$ of $80 \%$ aqueous ethanol solution. After mixing completely in a vortex, $100 \mu \mathrm{L}$ of each solution was used to react with $3 \mathrm{~mL}$ DPPH solution $(0.1 \mathrm{mM})$ for $30 \mathrm{~min}$ in the dark. The absorbance was obtained via a UV spectrophotometer at an absorbance of $516 \mathrm{~nm}$. The antioxidant activity of the fibrous membranes was expressed as a percentage of DPPH radical inhibition (\%AA) according to the following equation:

$$
\% \mathrm{AA}=\frac{\mathrm{A} \text { control }-\mathrm{A} \text { sample }}{\mathrm{A} \text { control }} \times 100 \%
$$

where $\mathrm{A}_{\text {control }}$ and $\mathrm{A}_{\text {sample }}$ represent blank absorbance and the values of the DPPH solution combined with the sample for each reaction, respectively. Experiments were tested in triplicate.

\subsection{Statistical Analysis}

The results were acquired using SPSS Statistics 22 software (IBM, Amun, NY, USA). One-way ANOVA with Tukey's multiple comparisons test $(p<0.05)$ was carried out to analyze the significance of fiber diameter, incorporation efficiency of TA, and antioxidant activity.

\section{Results and Discussion}

\subsection{Viscosity and Spinnability of GG Solutions}

TA is a non-polymeric functional molecule. According to our previous experiment [31], TA did not have any fiber-forming ability within the required concentration range because of its relatively low viscosity. In this work, natural polymer GG was used as the structure-forming matrix to avoid the breakup of the fluid jet into droplets during electrospinning. Despite previous report that GG solutions have fiber-forming ability [25], the scope to form the fibers had not yet been investigated. Therefore, the relationship between the rheology and the spinnability of GG was preferentially explored to find optimal concentration to load TA under stable electrospinning process.

As depicted in Figure 1, the apparent viscosity of GG solutions at each shear rate strongly depended on its concentration, in general increasing as concentration increased. The flow curves were carried out at $50{ }^{\circ} \mathrm{C}$. In all cases, GG solutions exhibited typical non-Newtonian (pseudoplastic) behavior with increasing shear rate $\left(>1 \mathrm{~s}^{-1}\right)$, in which the shear viscosity reduced with shear rate and became noticeable with increasing GG concentration, displaying shear thinning property. Similar results were reported by Martín-Alfonso [38] and Torres et al. [34]. Shear thinning is often explained to be the result of breakdown of interchain interactions in the polymer network, i.e. random movement of polymer chains become more progressively limited as the concentration increases and consequently the rate to reform new entanglements is lower than those unwinding under high shear stress, resulting in a decrease interaction in the polymer network as the concentration increases $[39,40]$.

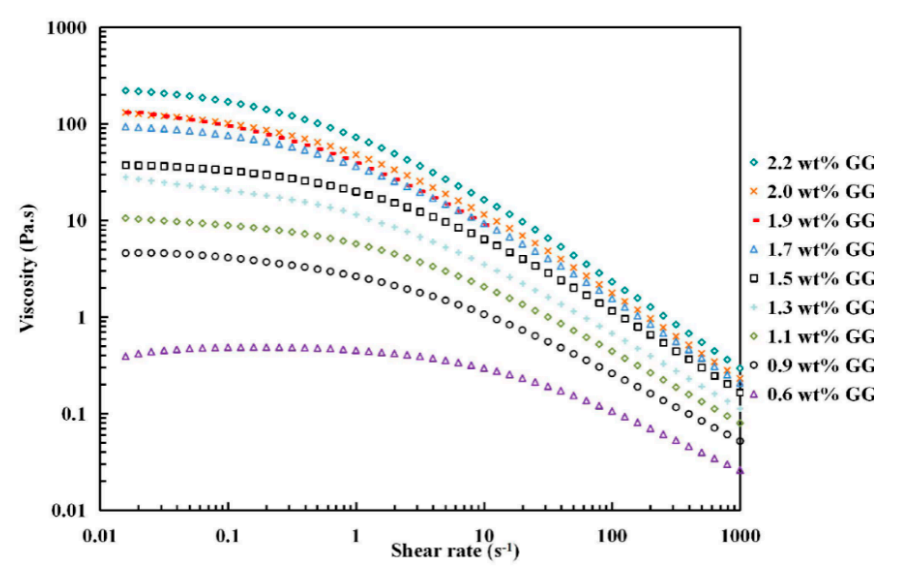

Figure 1. Apparent viscosity of GG solutions of different concentrations as a function of shear rate. 
Flow curves of GG solutions mentioned above showed a satisfactory fitting with the Cross-Williamson model $\left(R^{2}>0.99\right)$ in the shear rate range and the resultant zero-shear viscosities $\eta_{0}$ were used to further obtain specific viscosity values. By using the specific viscosity, chain entanglement concentration Ce was calculated from the plots of specific viscosity $\eta_{\text {sp }}$ data versus the GG concentration in double logarithmic coordinate for these polysaccharide solutions [35,41,42]. In electrospinning process, $\mathrm{Ce}$ is the minimum concentration before attaining stable electrospinning, with possible fabrication of uniform bead-free nanofibers [43,44]. As shown in Figure 2, the Ce was determined to be $\sim 1.1 \mathrm{wt} \%$ for the GG solution from the crossover of two different linear intervals. At low concentrations, specific viscosity $\eta_{\mathrm{sp}} \sim \mathrm{c}^{6.4}$ was observed, indicating GG solutions in this range were in semi-dilute unentangled state, in which the chain overlap between individual molecules was insufficient to form any notable degree of entanglement. Above $\mathrm{Ce}$, a notable increase in gradient was obtained and reached a constant slope of 8.10, suggesting the entrance of the semi-dilute entangled regime. The Ce detected in this study was slightly higher than Torres's report, where GG concentrations between 5 and $10 \mathrm{~g} / \mathrm{L}$ were suggested to be in the entangled regime when the test was carried out at $20^{\circ} \mathrm{C}$. The variation of Ce might due to the difference of sample temperature under test.

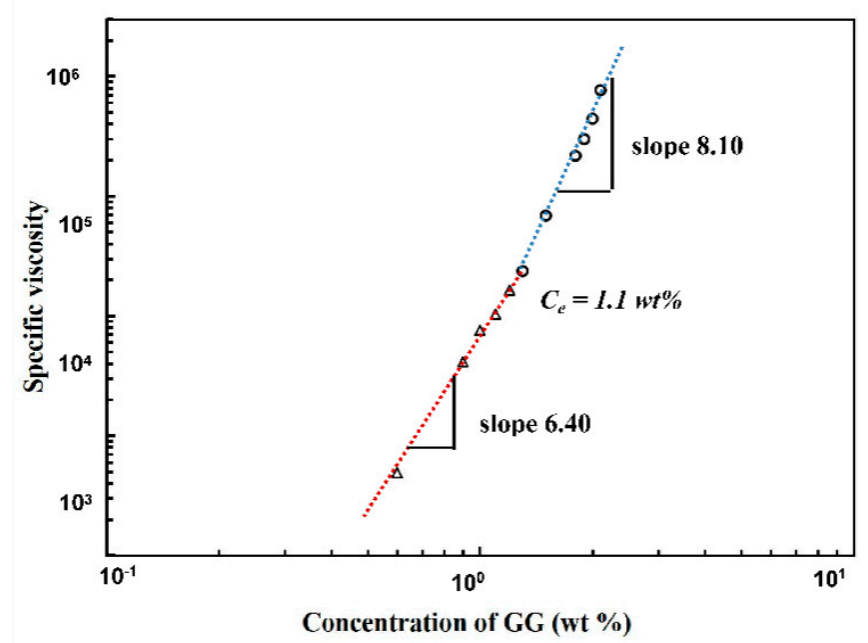

Figure 2. Plot of specific viscosity versus aqueous GG concentration.

\subsection{Morphology of Electrospun GG Fibers}

A series of GG solutions above the critical entanglement concentration (1.1 wt \% GG) were electrospun. During the spinning, a continuous jet with stable whipping movements could eject from the spinneret at the concentration range between 1.1 and $2.2 \mathrm{wt} \%$, allowing orderly deposit of fibers on the collector. However, it was difficult to continuously spin GG solutions when concentration was raised above $2.5 \mathrm{wt} \%$ since the solutions became too viscous, in which gel formed on the tip of needle and electro-spraying constantly occurred. The representative microscopic images of the collected fibers were examined by SEM, as shown in Figure 3. At entanglement concentration $(1.1 \mathrm{wt} \%)$, the resulting electrospun fibers consisted of more beads with limited incipient fibers (Figure 3A). The formation of GG fibers became obvious when the concentration roswas raised to $1.5 \mathrm{wt} \%$, but most fibers exhibited a beaded-on-string morphology (Figure 3C). The transition from beaded-on-string structure to defect-free fibers occurred at $2 \mathrm{wt} \%$ of GG solution, which was the minimum threshold that yielded smooth and uniform microstructure (Figure 3F), indicating molecules of GG exhibit enough intermolecular force to stable extensional deformation caused by the applied electric filed. Experimentally, the concentrations to obtain defect-free or beadless fibers here were found at 1.8-2.3 times Ce, which was close to that of other biopolymer solutions in previous reports $[6,35,45]$. 

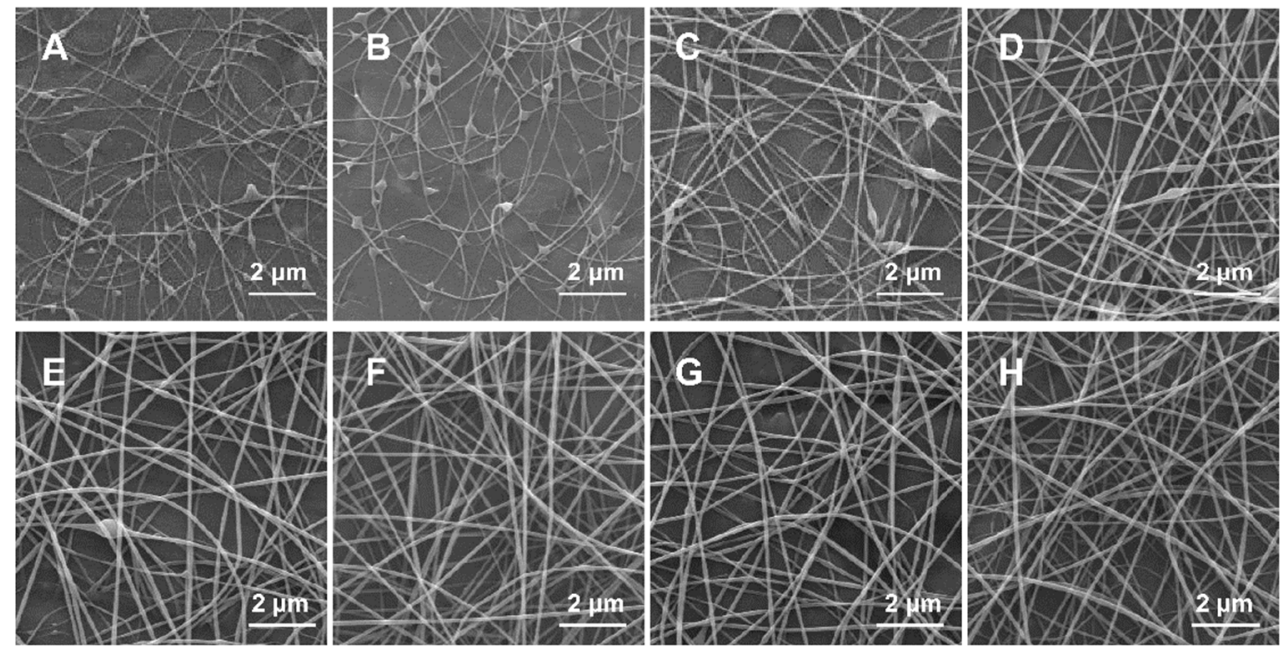

Figure 3. SEM images of electrospun fibers obtained from GG solutions with concentration of: (A) 1.1 wt \%; (B) $1.3 \mathrm{wt} \%$; (C) $1.5 \mathrm{wt} \%$; (D) $1.7 \mathrm{wt} \%$; (E) $1.9 \mathrm{wt} \%$; (F) $2.0 \mathrm{wt} \%$; (G) $2.1 \mathrm{wt} \%$; and (H) $2.2 \mathrm{wt} \%$. Magnification is $10,000 \times$.

The homogeneous morphology of GG obtained from purified GG solution had different results in some aspects from Lubambo's results [25]. In particular, the uniform fibers could be obtained in a more simplified process without using any filtration procedure. This disparity might result from the preparation method, detailed purification process of the solution and parameters of electrospinning.

\subsection{Effect of Incorporating TA on the Morphology of GG-TA Nanofibers}

In blended GG-TA solutions, 2 wt \% of GG solution was chosen as the carrier to incorporate different loading amount of natural antioxidant TA. Fiber forming ability of GG-TA solutions was explored as a function of TA concentration under the same spinning parameters with GG. As depicted in Figure 4, it was determined that inclusion of TA has a dramatic effect on the morphology of the fibers as well as the fiber diameter distribution. The average diameter size ranged from 96 to $188 \mathrm{~nm}$ and followed non-normal distributions $(p<0.05)$. The incorporation of TA did not produce visible defects in the GG fibers when the TA increased from 5 to $10 \mathrm{wt} \%$ (compared Figure 4A,B), while obvious aggregation formed as the concentration of TA increased to $15 \mathrm{wt} \%$ (Figure $4 \mathrm{C}$ ). In addition, the average diameter increased from 138 to $188 \mathrm{~nm}$ as the concentration of TA increased from 15 to 20 wt $\%(p<0.05$; Figure 4D) in comparison to $10 \mathrm{wt} \%$ (Figure 4B). Stable electrospinning could be carried out for several hours to finally obtain fibrous mats. It is worth noting that the formation of nanofibers in this system was manufactured using water as the solvent without using any synthetic chemicals. These results are encouraging findings for green electrospinning and its potential food-grade applications. 

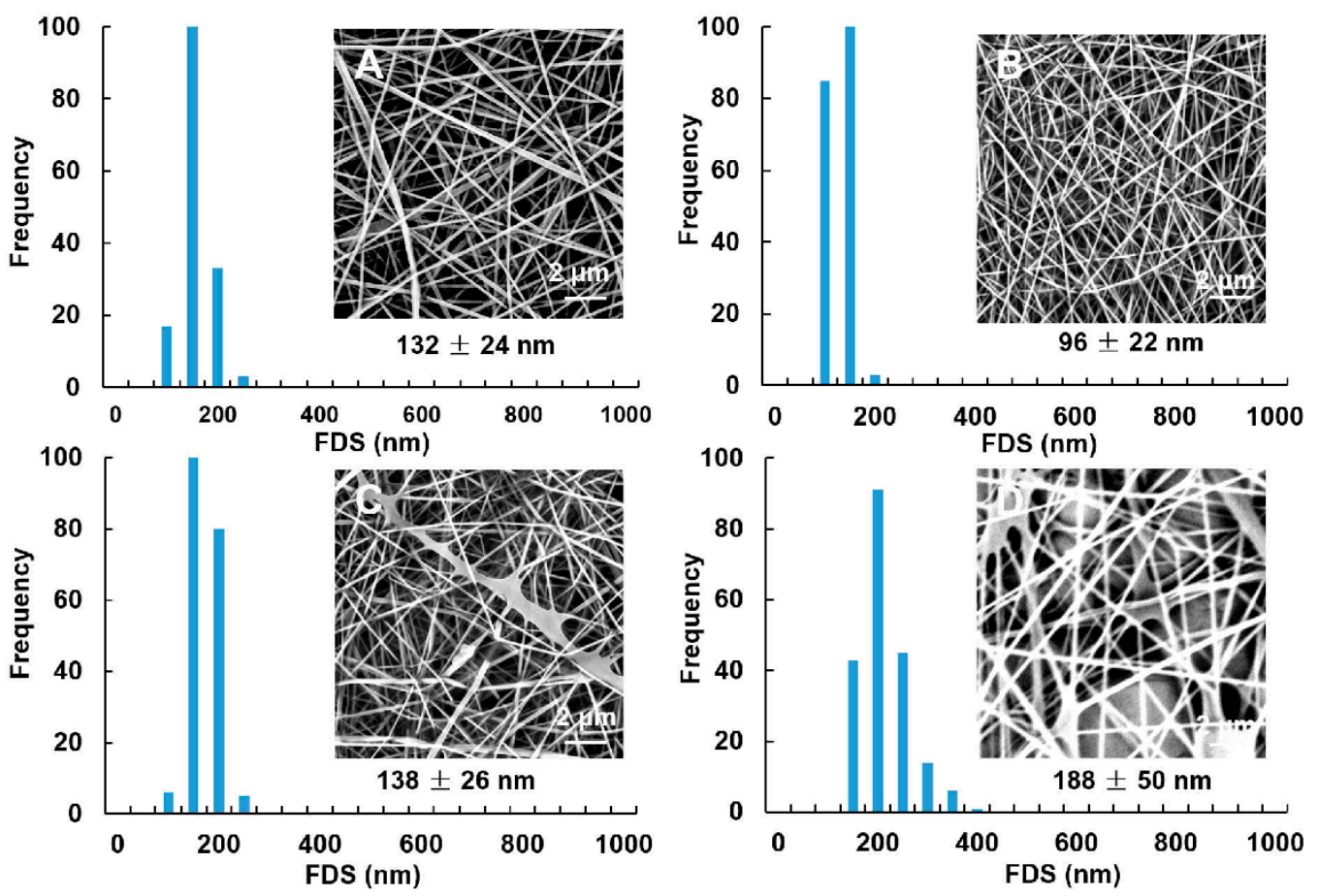

Figure 4. SEM images and fiber diameter size (FDS) of electrospun fibers obtained from GG solutions at 2 wt \% concentration loaded with: 5 wt \% TA (A); 10 wt \% TA (B); 15 wt \% TA (C); and 20 wt \% TA (D). Magnification is $10,000 \times$.

\subsection{Loading Efficiency of $T A$}

Our pretests using the spectrophotometer have shown that both the TA standard and the fibrous mats loaded with TA have their maximum absorbance observed at $275 \mathrm{~nm}$ [31], while neat GG fibrous mats showed no absorption in this range. The loading efficiency of TA was calculated from the linear regression equation of the standard curve, as listed in Table 1. The results confirm the existence of TA in fibrous mats and show that all of the GG-TA fibrous mats had high loading efficiency, close to $100 \%$. Another research group obtained similar results, finding that the loading efficiency for gallic acid varied from $99 \%$ to $105 \%$ in a zein-gallic acid electrospun fibers system [11]. These results suggest that the electrospinning technique is feasible for the given delivery system and can efficiently incorporate instructive molecules to produce functional micro- to nanoscale fibrous mats. Encapsulation of this food-grade antioxidant in the nano-scale delivery systems may exhibit a unique structure compared with macro antioxidants, potentially applying in prolong shelf life of foods as edible packaging or functional foods.

Table 1. Incorporation efficiency of GG fibrous mats loaded with TA.

\begin{tabular}{cccc}
\hline GG-TA fibrous mat & $\mathbf{1 0} \%$ TA & $\mathbf{1 5 \%}$ TA & $\mathbf{2 0 \%}$ TA \\
\hline Actual concentration $(\%)$ & $9.98 \pm 0.98^{\mathrm{a}}$ & $15.6 \pm 0.29^{\mathrm{b}}$ & $20.01 \pm 2.49^{\mathrm{c}}$ \\
Incorporation efficiency $(\%)$ & 99.8 & 100.4 & 100.0 \\
\hline
\end{tabular}

The data are expressed as mean \pm standard derivation. Different letters $(\mathrm{a}-\mathrm{c})$ represent the data on the same row are significantly different $(p<0.05)$.

\subsection{Interactions between TA and GG in GG-TA Fibrous Mats}

To assign intermolecular interactions between TA and GG, as-spun GG fibrous mats with and without incorporating TA were characterized using FT-IR spectra. In Figure 5, Curves a and b refer to the spectra of TA powders and neat GG fibrous mats, respectively, while Curves c-e represent the spectra of GG fibrous mats incorporated with 10, 15, and $20 \mathrm{wt} \% \mathrm{TA}$, respectively. The characteristic 
absorption peak of TA was identified using FT-IR examination (Figure 5, Curves c-e vs. Curve a) through the presence of $\mathrm{C}=\mathrm{O}$ stretching vibration of ester group $\left(1716 \mathrm{~cm}^{-1}\right)$, aromatic $-\mathrm{C}=\mathrm{C}$ - stretching vibration (1613 and $\left.1533 \mathrm{~cm}^{-1}\right)$, and $\mathrm{O}-\mathrm{H}$ stretching of alkylphenol $\left(1323 \mathrm{~cm}^{-1}\right)$, indicating the presence of TA loaded on electrospun GG fibrous mats.

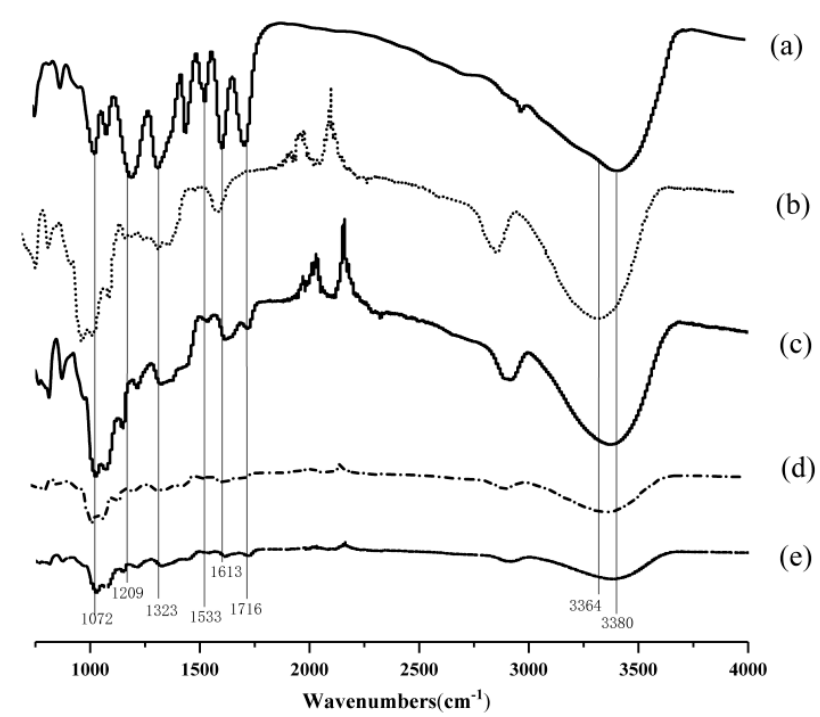

Figure 5. FT-IR spectra of the: TA powder (a); neat GG (b); and of GG fibrous mats loaded with: 10\% TA (c); $15 \%$ TA (d); and $20 \%$ TA (e).

The increase in TA led to a marked difference in the FT-IR spectra of the GG-TA fibrous mats. The broad peaks that occurred at 3364 and $3380 \mathrm{~cm}^{-1}$ were related to the stretching mode of interchain interaction via hydrogen bonding, and became weaker when TA increased from 10 to $20 \mathrm{wt} \%$ (Figure 5c-d). Additionally, the peaks at 1209 and $1323 \mathrm{~cm}^{-1}$, due to $\mathrm{O}-\mathrm{H}$ vibration from phenolic hydroxyl of TA, were less discernible in the fibers incorporated with TA when the TA increased to $15 \mathrm{wt} \%$ than with $10 \mathrm{wt} \%$ (Figure 5, Curve d vs. Curve c) and neat GG fibrous mats (Figure 5, Curve b). These peaks did not change noticeably with $20 \mathrm{wt} \%$ of TA loading. Similarly, another marked reduction appeared at $1072 \mathrm{~cm}^{-1}$ and correlated to $\mathrm{C}-\mathrm{O}$ stretching within the cyclic ether group of GG when TA increased from 10 to $15 \mathrm{wt} \%$. These observations suggest that a particular arrangement might exist in the fibers attributed to the intermolecular hydrogen bonding between TA and GG in the GG-TA matrix. From the FT-IR results, it is suggested that, after the addition of TA, new hydrogen bonds were formed between the phenolic hydroxyl of TA and hydroxyl and cyclic ether groups of the GG molecules, and thus occupied the functional group of GG matrix subsequently disrupting the existing hydrogen bonding in the GG. This was confirmed by the changes of viscosity after addition of TA to GG solutions, as shown in Table 2. These interactions are similar to the interactions in chitosan-based films for polyphenols of tea with chitosan [46]. The resultant interactions were maintained during electrospinning as $\mathrm{H}_{2} \mathrm{O}$ molecules evaporated, possibly in response to the alteration in fiber morphology previously shown in Figure 4.

Table 2. Viscosity of GG and various GG-TA solutions.

\begin{tabular}{ccccc}
\hline Sample & GG & GG-10\% TA & GG-15\% TA & GG-20\% TA \\
\hline Viscosity (Pa.s) & $204.41 \pm 23.2^{\mathrm{a}}$ & $270.58 \pm 43.5^{\mathrm{b}}$ & $290.23 \pm 45.2^{\mathrm{c}}$ & $312.08 \pm 52.6^{\mathrm{d}}$ \\
\hline
\end{tabular}

The data are expressed as mean \pm standard derivation. Different letters (a-d) represent the data on the same row are significantly different $(p<0.05)$. 


\subsection{XRD of GG-TA Fibrous Mats}

The crystallinity of GG and GG-TA fibrous mats after electrospinning, as tested by XRD, is shown in Figure 6. The broad peak observed in Figure 6 indicates that neat GG exhibited low viscosity. Similar results are reported for native GG solutions [47,48]. The shape and position of the GG-TA 10\% (Figure 6, Curve b), GG-TA 15\% (Figure 6, Curve c), and GG-TA 20\% (Figure 6, Curve d) peaks did not change when compared to the neat GG fibrous mats, which suggested that TA does not have a distinct effect on the crystallinity property of GG.

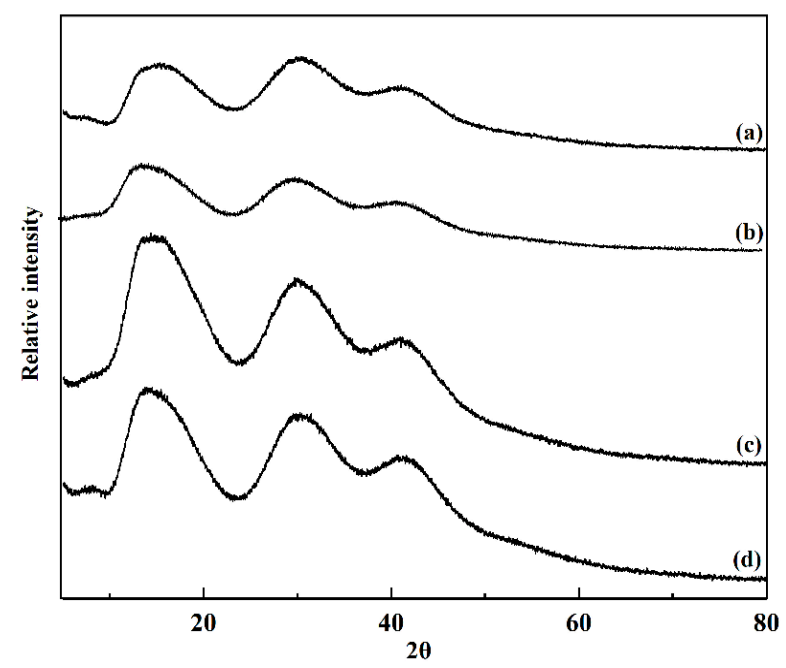

Figure 6. XRD study of: the neat GG (a); and of the GG fibrous mats loaded with TA with: 10\% TA (b); $15 \%$ TA (c); and 20\% TA (d).

As demonstrated in the XRD profile (Figure 6, Curves a-c), the intensity of peaks increased proportionally as TA concentration increased from 10 to $15 \mathrm{wt} \%$, indicating that intensity in the diffraction pattern of the GG-TA fibrous mats was strongly influenced by the presence of TA. It is worth mentioning that the diffraction peak unexpectedly decreased with higher levels of TA content (Figure 6, Curves $\mathrm{c}$ and $\mathrm{d}$ ). Based on the FT-IR results, it can be assumed that hydrogen bonding between TA and GG may drive the TA molecules to gradually disperse into the GG matrix, thus interrupting the original crystal structure between GG and TA. Moreover, these two components have better miscibility at $20 \mathrm{wt} \%$ TA than at $15 \mathrm{wt} \%$ TA. A similar observation of a konjac glucomannan/curdlan blended film is previously reported with the increased addition of konjac glucomannan in the blends [49]. The XRD results show good agreement with the obtained fiber morphology, where more aggregates formed in GG-TA 15\% while improved morphology was obtained in GG-TA 20\%.

\subsection{Thermal Stability of GG-TA Fibrous Mats}

The thermal behavior of neat GG and GG-TA fibrous mats was investigated by DSC method, as depicted in Figure 7, and GG powder exhibited two endothermic peaks (278 and $318{ }^{\circ} \mathrm{C}$ ), which correspond to similar thermograms that show the peaks occurring at $257.81-261.95{ }^{\circ} \mathrm{C}$ and 302.46-311.57 ${ }^{\circ} \mathrm{C}$ (Figure 7, Curve a), as reported by Razmkhah [50]. However, the neat GG fibrous mat exhibited only one exothermic peak $\left(298^{\circ} \mathrm{C}\right)$. This was attributed to the orientation effect, due to the high speed elongation and rapid solidification during electrospinning [51] (Figure 7, Curve b). All of the GG-TA fibrous mats showed a major degradation curve at around $300^{\circ} \mathrm{C}$ (Figure 7, Curves a-c), which can be explained as the result of the disintegration of the polysaccharide backbone. 


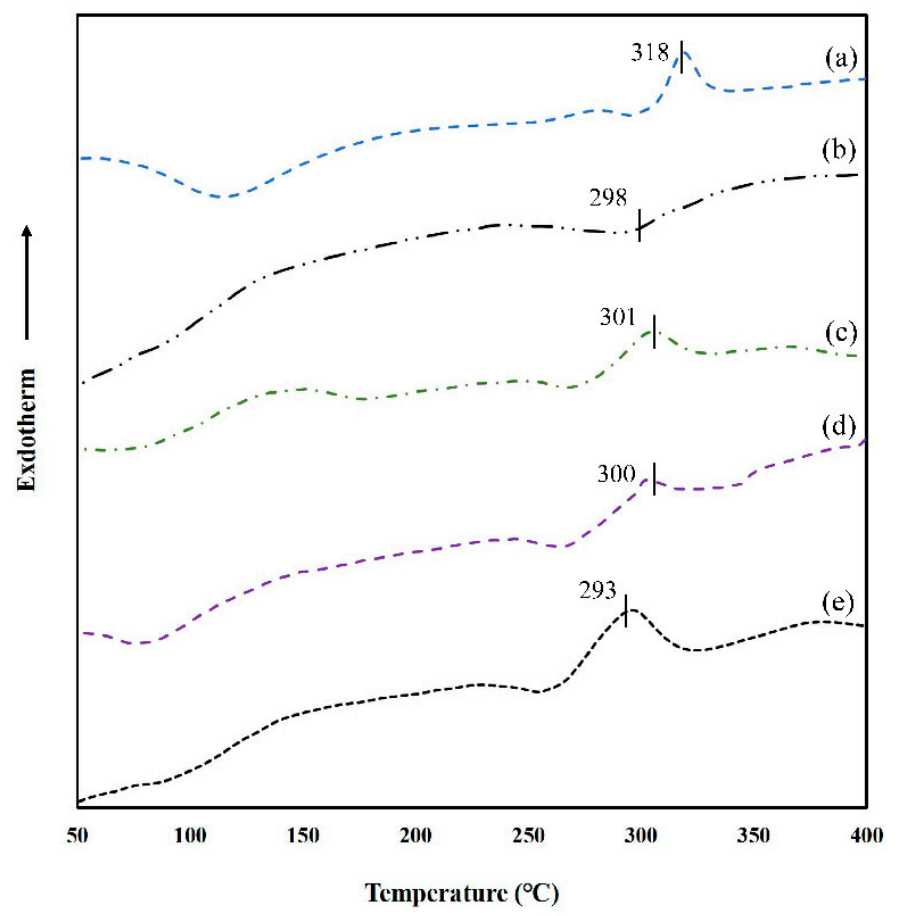

Figure 7. DSC thermograms of: GG powder (a); neat GG (b); and of GG fibrous mats loaded with: $10 \%$ TA (c); $15 \%$ TA (d); and $20 \%$ TA (e).

Increasing the TA concentration resulted in a slight decrease to the decomposition Tm. Taking FT-IR results into account, this was attributed to the deformation of the strong H-bond network in the phenolic hydroxyl of TA, and hydroxyl and cyclic ether groups of GG molecules. In agreement with the Tm reduction, $\Delta \mathrm{Hm}$ shifted to lower values when TA increased from 10 to $15 \mathrm{wt} \%$ (Table 3 ). In addition, the apparent melting enthalpy values increased from 37.53 to $122.1 \mathrm{~J} / \mathrm{g}$ when TA concentration was further raised from 15 to $20 \mathrm{wt} \%$, whereas the opposite trend was found for the Tm of the network. This phenomenon suggests that the greater is the amount of TA introduced into the GG matrix, the higher is the density of the ordered junction zones formed, as a result that higher enthalpy is required to disrupt inter-chain interactions, due to the dense network formed and limited segmental mobility of polymer chains. Thermal results suggest that GG-TA fibrous mats seems to be an attractive option as an edible antioxidant film for preserving any processed food which could be produced at a temperature lower than $290^{\circ} \mathrm{C}$.

Table 3. Thermodynamic properties of the neat GG and GG fibrous mats loaded with TA.

\begin{tabular}{cccccc}
\hline Sample & GG-powder & GG-fiber & $\mathbf{1 0} \%$ TA & $\mathbf{1 5 \%}$ TA & $\mathbf{2 0 \%}$ TA \\
\hline $\operatorname{Tm}\left({ }^{\circ} \mathrm{C}\right)$ & 318 & 298 & 301 & 300 & 293 \\
$\Delta \mathrm{H}(\mathrm{J} / \mathrm{g})$ & 47.76 & 82.14 & 49.68 & 37.53 & 122.10 \\
\hline
\end{tabular}

\subsection{Antioxidant Activity of GG-TA Fibrous Mats}

The antioxidant properties of the fibrous mats were evaluated by DPPH tests and the results, representing DPPH scavenging activity, are depicted in Figure 8. These results indicate that TA maintains inhibition activity after blending and being electrospun with GG. The DPPH scavenging activity of the TA loaded GG electrospun membranes changed from $16.6 \%$ to $80 \%$ when TA concentration increased from $5 \%$ to $20 \%$, while the neat GG fibrous mat only exhibited a weak influence $(3.5 \%$ of inhibition) on the DPPH solutions. A 22 times enhancement was observed at $20 \mathrm{wt} \%$ TA loading in contrast with the control sample of GG without TA $(p<0.05)$, indicating a positive correlation between the content of loaded TA and the increase in the DPPH scavenging capacity. 


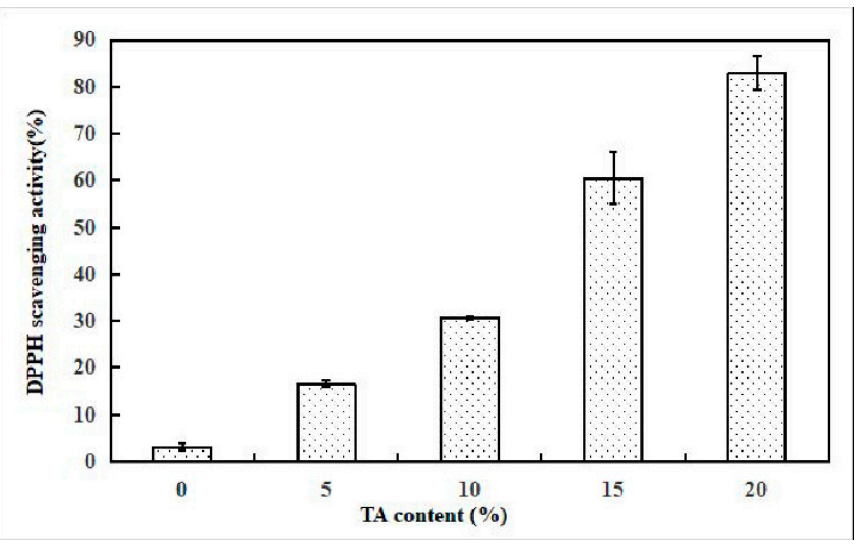

Figure 8. Antioxidant activity of the neat GG and of GG fibrous mats loaded with TA.

\section{Conclusions}

This is the first report demonstrating the feasibility of using natural polysaccharide guar gum (GG) to develop electrospun GG fibrous mats with antioxidant activity without using any synthetic chemicals. The correlation between GG solution properties and the morphology of GG nanofibers was investigated prior to electrospinning. This work clearly shows that the incorporation of different ratio of TA into the GG fibrous mats leads to dramatic effects on the composite fiber morphology. Intermolecular interaction between TA and GG were confirmed to be hydrogen bonding by FT-IR, and the crystallinity and thermal stability showed varied changes accordingly. Fibrous mats loaded with TA provided excellent antioxidant activity. The characterizations presented here will aid future research in the optimization of GG-TA solution for electrospinning, potentially broadening the development of edible active packaging and delivery of therapeutics.

Author Contributions: X.L., W.Y., M.Z., L.S.L. and A.M.M.S. designed the experiments. W.Y. wrote the manuscript. W.Y., J.J., and Q.Z. performed the experiments and analyzed the data. S.P. contributed to the language editing.

Funding: This work was supported by the National Key R \& D project (2018YFD0401300), Natural Science Foundation of Tianjin (No:18JCQNJC14600) and Tianjin Sci-Tech Project (18ZYPTJC00020).

Acknowledgments: The authors thank Juan Zhao (from the Tianjin University of Science and Technology, Tianjin, China) for the technical assistance in the FT-IR, He Wang and Fei Wang for the technical assistance in the electrospinning (Nankai University, Tianjin, China), and Xianai Shi (Fuzhou University, Fujian, China) for the design of experiments.

Conflicts of Interest: The authors declare no conflict of interest.

\section{References}

1. Wenguo, C.; Yue, Z.; Jiang, C. Electrospun nanofibrous materials for tissue engineering and drug delivery. Sci. Technol. Adv. Mater. 2010, 11, 014108.

2. Sahay, R.; Kumar, P.S.; Sridhar, R.; Sundaramurthy, J.; Venugopal, J.; Mhaisalkar, S.G.; Ramakrishna, S. Electrospun composite nanofibers and their multifaceted applications. J. Mater. Chem. 2012, 22, 12953-12971. [CrossRef]

3. Horcajada, P.; Chalati, T.; Serre, C.; Gillet, B.; Sebrie, C.; Baati, T.; Eubank, J.F.; Heurtaux, D.; Clayette, P.; Kreuz, C.; et al. Porous metal-Organic-framework nanoscale carriers as a potential platform for drug delivery and imaging. Nat Mater. 2009, 9, 172. [CrossRef]

4. Huang, Z.-M.; Zhang, Y.Z.; Kotaki, M.; Ramakrishna, S. A review on polymer nanofibers by electrospinning and their applications in nanocomposites. Compos. Sci. Technol. 2003, 63, 2223-2253. [CrossRef]

5. Jordan, A.M.; Viswanath, V.; Kim, S.-E.; Pokorski, J.K.; Korley, L.T.J. Processing and surface modification of polymer nanofibers for biological scaffolds: A review. Compos. Sci. Technol. B 2016, 4, 5958-5974. [CrossRef] 
6. Rieger, K.A.; Birch, N.P.; Schiffman, J.D. Electrospinning chitosan/poly (ethylene oxide) solutions with essential oils: Correlating solution rheology to nanofiber formation. Carbohydr. Polym. 2016, 139, 131-138. [CrossRef]

7. Sill, T.J.; von Recum, H.A. Electrospinning: Applications in drug delivery and tissue engineering. Biomaterials 2008, 29, 1989-2006. [CrossRef]

8. Ghorani, B.; Tucker, N. Fundamentals of electrospinning as a novel delivery vehicle for bioactive compounds in food nanotechnology. Food Hydrocoll. 2015, 51, 227-240. [CrossRef]

9. Paaver, U.; Heinämäki, J.; Laidmäe, I.; Lust, A.; Kozlova, J.; Sillaste, E.; Kirsimäe, K.; Veski, P.; Kogermann, K. Electrospun nanofibers as a potential controlled-release solid dispersion system for poorly water-soluble drugs. Int. J. Pharm. 2015, 479, 252-260. [CrossRef]

10. Luo, Y.; Zhang, B.; Whent, M.; Yu, L.; Wang, Q. Preparation and characterization of zein/chitosan complex for encapsulation of $\alpha$-tocopherol, and its in vitro controlled release study. Colloids Surf. B Biointerfaces 2011, 85, 145-152. [CrossRef]

11. Neo, Y.P.; Ray, S.; Jin, J.; Gizdavic-Nikolaidis, M.; Nieuwoudt, M.K.; Liu, D.; Quek, S.Y. Encapsulation of food grade antioxidant in natural biopolymer by electrospinning technique: A physicochemical study based on zein-gallic acid system. Food Chem. 2013, 136, 1013-1021. [CrossRef] [PubMed]

12. Oehlke, K.; Adamiuk, M.; Behsnilian, D.; Gräf, V.; Mayer-Miebach, E.; Walz, E.; Greiner, R. Potential bioavailability enhancement of bioactive compounds using food-grade engineered nanomaterials: A review of the existing evidence. Food Funct. 2014, 5, 1341-1359. [CrossRef] [PubMed]

13. Mine, S.; Prasad, K.; Izawa, H.; Sonoda, K.; Kadokawa, J.-I. Preparation of guar gum-based functional materials using ionic liquid. J. Mater. Chem. 2010, 20, 9220-9225. [CrossRef]

14. Verger, L.; Corre, S.; Poirot, R.; Quintard, G.; Fleury, E.; Charlot, A. Dual guar/ionic liquid gels and biohybrid material thereof: Rheological investigation. Carbohydr. Polym. 2014, 102, 932-940. [CrossRef]

15. Mudgil, D.; Barak, S.; Khatkar, B.S. Guar gum: Processing, properties and food applications-A Review. J. Food Sci. Technol. 2014, 51, 409-418. [CrossRef] [PubMed]

16. Zheng, M.; You, Q.; Lin, Y.; Lan, F.; Luo, M.; Zeng, H.; Zheng, B.; Zhang, Y. Effect of guar gum on the physicochemical properties and in vitro digestibility of lotus seed starch. Food Chem. 2019, 272, $286-291$. [CrossRef]

17. Sharma, G.; Sharma, S.; Kumar, A.; Al-Muhtaseb, A.a.H.; Naushad, M.; Ghfar, A.A.; Mola, G.T.; Stadler, F.J. Guar gum and its composites as potential materials for diverse applications: A review. Carbohydr. Polym. 2018, 199, 534-545. [CrossRef] [PubMed]

18. Krishnaiah, Y.S.R.; Satyanarayana, S.; Rama Prasad, Y.V.; Narasimha Rao, S. Gamma scintigraphic studies on guar gum matrix tablets for colonic drug delivery in healthy human volunteers. J. Control Release 1998, 55, 245-252. [CrossRef]

19. Ghaffarzadegan, T.; Marungruang, N.; Fåk, F.; Nyman, M. Molecular Properties of Guar Gum and Pectin Modify Cecal Bile Acids, Microbiota, and Plasma Lipopolysaccharide-Binding Protein in Rats. PLoS ONE 2016, 11, e0157427. [CrossRef]

20. McRorie, J.W.; McKeown, N.M. Understanding the Physics of Functional Fibers in the Gastrointestinal Tract: An Evidence-Based Approach to Resolving Enduring Misconceptions about Insoluble and Soluble Fiber. J. Acad. Nutr. Diet. 2017, 117, 251-264. [CrossRef]

21. Kumar, V.S.; Rijo, J.; Sabitha, M. Guargum and Eudragit ${ }^{\circledR}$ coated curcumin liquid solid tablets for colon specific drug delivery. Int. J. Biol. Macromol. 2018, 110, 318-327. [CrossRef]

22. Krishnaiah, Y.S.R.; Satyanarayana, V.; Dinesh Kumar, B.; Karthikeyan, R.S. In vitro drug release studies on guar gum-based colon targeted oral drug delivery systems of 5-fluorouracil. Eur. J. Pharm. Sci. 2002, 16, 185-192. [CrossRef]

23. Praphakar, R.A.; Jeyaraj, M.; Mehnath, S.; Higuchi, A.; Ponnamma, D.; Sadasivuni, K.K.; Rajan, M. A pH-sensitive guar gum-grafted-lysine- $\beta$-cyclodextrin drug carrier for the controlled release of 5 -flourouracil into cancer cells. J. Mater. Chem. B 2018, 6, 1519-1530. [CrossRef]

24. Doyle, J.; Lyons, G.; Morris, E. New proposals on “hyperentanglement” of galactomannans: Solution viscosity of fenugreek gum under neutral and alkaline conditions. Food Hydrocoll. 2009, 23, 1501-1510. [CrossRef]

25. Lubambo, A.F.; de Freitas, R.A.; Sierakowski, M.-R.; Lucyszyn, N.; Sassaki, G.L.; Serafim, B.M.; Saul, C.K. Electrospinning of commercial guar-gum: Effects of purification and filtration. Carbohydr. Polym. 2013, 93, 484-491. [CrossRef] 
26. Rezaei, A.; Nasirpour, A. Characterization and water solubility of curcumin/cyclodextrin inclusion complex-loaded almond gum/PVA electrospun nanofibers. In Proceedings of the 7 th International Conference on Nanostructures (ICNS7), Tehran, Iran, 27 February-1 March 2018.

27. Rezaei, A.; Tavanai, H.; Nasirpour, A. Fabrication of electrospun almond gum/PVA nanofibers as a thermostable delivery system for vanillin. Int. J. Biol. Macromol. 2016, 91, 536-543. [CrossRef] [PubMed]

28. Gülçin, İ.; Huyut, Z.; Elmastaş, M.; Aboul-Enein, H.Y. Radical scavenging and antioxidant activity of tannic acid. Arab. J. Chem. 2010, 3, 43-53. [CrossRef]

29. Jordan, L.G.; Booth, B.W. HER ${ }^{2+}$ breast cancer cells undergo apoptosis upon exposure to tannic acid released from remodeled cross-linked collagen type I. J. Biomed. Mater. Res. Part A 2018, 106, 26-32. [CrossRef]

30. Pyla, R.; Kim, T.-J.; Silva, J.L.; Jung, Y.-S. Enhanced antimicrobial activity of starch-based film impregnated with thermally processed tannic acid, a strong antioxidant. Int. J. Food Microbiol. 2010, 137, $154-160$. [CrossRef]

31. Yang, W.; Sousa, A.M.M.; Fan, X.; Jin, T.; Li, X.; Tomasula, P.M.; Liu, L. Electrospun ultra-fine cellulose acetate fibrous mats containing tannic acid-Fe ${ }^{3+}$ complexes. Carbohydr. Polym. 2017, 157, 1173-1179. [CrossRef]

32. Fathi, M.; Martín, Á.; McClements, D.J. Nanoencapsulation of food ingredients using carbohydrate based delivery systems. Trends Food Sci. Technol. 2014, 39, 18-39. [CrossRef]

33. Anu Bhushani, J.; Anandharamakrishnan, C. Electrospinning and electrospraying techniques: Potential food based applications. Trends Food Sci. Technol. 2014, 38, 21-33. [CrossRef]

34. Torres, M.D.; Hallmark, B.; Wilson, D.I. Effect of concentration on shear and extensional rheology of guar gum solutions. Food Hydrocoll. 2014, 40, 85-95. [CrossRef]

35. Kong, L.; Ziegler, G.R. Role of Molecular Entanglements in Starch Fiber Formation by Electrospinning. Biomacromolecules 2012, 13, 2247-2253. [CrossRef] [PubMed]

36. Li, X.; Chen, H.; Yang, B. Centrifugally spun starch-based fibers from amylopectin rich starches. Carbohydr. Polym. 2016, 137, 459-465. [CrossRef] [PubMed]

37. Yang, W.; Sousa, A.M.M.; Thomas-Gahring, A.; Fan, X.; Jin, T.; Li, X.; Tomasula, P.M.; Liu, L. Electrospun Polymer Nanofibers Reinforced by Tannic Acid/Fe ${ }^{+++}$Complexes. Materials 2016, 9, 757. [CrossRef]

38. Martín-Alfonso, J.E.; Cuadri, A.A.; Berta, M.; Stading, M. Relation between concentration and shear-extensional rheology properties of xanthan and guar gum solutions. Carbohydr. Polym. 2018, 181, 63-70. [CrossRef]

39. Bourbon, A.I.; Pinheiro, A.C.; Ribeiro, C.; Miranda, C.; Maia, J.M.; Teixeira, J.A.; Vicente, A.A. Characterization of galactomannans extracted from seeds of Gleditsia triacanthos and Sophora japonica through shear and extensional rheology: Comparison with guar gum and locust bean gum. Food Hydrocoll. 2010, 24, 184-192. [CrossRef]

40. Duxenneuner, M.R.; Fischer, P.; Windhab, E.J.; Cooper-White, J.J. Extensional Properties of Hydroxypropyl Ether Guar Gum Solutions. Biomacromolecules 2008, 9, 2989-2996. [CrossRef]

41. Wang, C.; Wang, Y.; Hashimoto, T. Impact of Entanglement Density on Solution Electrospinning: A Phenomenological Model for Fiber Diameter. Macromolecules 2016, 49, 7985-7996. [CrossRef]

42. Wang, C.; Hashimoto, T. Self-Organization in Electrospun Polymer Solutions: From Dissipative Structures to Ordered Fiber Structures through Fluctuations. Macromolecules 2018, 51, 4502-4515. [CrossRef]

43. Hodgkinson, T.; Chen, Y.; Bayat, A.; Yuan, X.-F. Rheology and Electrospinning of Regenerated Bombyx mori Silk Fibroin Aqueous Solutions. Biomacromolecules 2014, 15, 1288-1298. [CrossRef] [PubMed]

44. Tiwari, S.K.; Venkatraman, S.S. Importance of viscosity parameters in electrospinning: Of monolithic and core-shell fibers. Mater. Sci. Eng. C 2012, 32, 1037-1042. [CrossRef]

45. McKee, M.G.; Wilkes, G.L.; Colby, R.H.; Long, T.E. Correlations of Solution Rheology with Electrospun Fiber Formation of Linear and Branched Polyesters. Macromolecules 2004, 37, 1760-1767. [CrossRef]

46. Siripatrawan, U.; Harte, B.R. Physical properties and antioxidant activity of an active film from chitosan incorporated with green tea extract. Food Hydrocoll. 2010, 24, 770-775. [CrossRef]

47. Cunha, P.L.R.; Castro, R.R.; Rocha, F.A.C.; de Paula, R.C.M.; Feitosa, J.P.A. Low viscosity hydrogel of guar gum: Preparation and physicochemical characterization. Int. J. Biol. Macromol. 2005, 37, 99-104. [CrossRef]

48. Li, N.; Liu, C.; Chen, W. Facile Access to Guar Gum Based Supramolecular Hydrogels with Rapid Self-Healing Ability and Multistimuli Responsive Gel-Sol Transitions. J. Agric. Food Chem. 2018. [CrossRef] [PubMed] 
49. Wu, C.; Peng, S.; Wen, C.; Wang, X.; Fan, L.; Deng, R.; Pang, J. Structural characterization and properties of konjac glucomannan/curdlan blend films. Carbohydr. Polym. 2012, 89, 497-503. [CrossRef] [PubMed]

50. Razmkhah, S.; Mohammadifar, M.A.; Razavi, S.M.A.; Ale, M.T. Purification of cress seed (Lepidium sativum) gum: Physicochemical characterization and functional properties. Carbohydr. Polym. 2016, 141, 166-174. [CrossRef] [PubMed]

51. Rustemkyzy, C.; Belton, P.; Qi, S. Preparation and Characterization of Ultrarapidly Dissolving Orodispersible Films for Treating and Preventing Iodine Deficiency in the Pediatric Population. J. Agric. Food Chem. 2015, 63, 9831-9838. [CrossRef] [PubMed]

(C) 2019 by the authors. Licensee MDPI, Basel, Switzerland. This article is an open access article distributed under the terms and conditions of the Creative Commons Attribution (CC BY) license (http://creativecommons.org/licenses/by/4.0/). 